\title{
Diacronie
}

Studi di Storia Contemporanea

$\mathrm{N}^{\circ} 21,1$ | 2015

Le città di Babele

\section{Alessandra Coppola, Una faccia una razza? Grecia antica e moderna nell'immaginario italiano di età fascista}

Jacopo Bassi

\section{(2) OpenEdition}

Journals

\section{Edizione digitale}

URL: http://journals.openedition.org/diacronie/1932

DOI: 10.4000/diacronie.1932

ISSN: 2038-0925

Editore

Association culturelle Diacronie

Notizia bibliografica digitale

Jacopo Bassi, « Alessandra Coppola, Una faccia una razza? Grecia antica e moderna nell'immaginario italiano di età fascista », Diacronie [Online], № 21, 1 | 2015, documento 11, Messo online il 01 mars 2015, consultato il 22 septembre 2020. URL : http://journals.openedition.org/diacronie/1932 ; DOI : https://doi.org/10.4000/diacronie.1932 


\section{Diacronie}

\section{RECENSIONE:}

\section{Alessandra COPPOLA, Una faccia una razza? Grecia antica e moderna nell'immaginario italiano di età fascista, Roma, Carocci, 2013, 168 pp.}

a cura di Jacopo BASSI *

«Una faccia una razza» è un'espressione che racchiude in sé la complessità dei rapporti fra Grecia e Italia; il titolo del libro di Alessandra Coppola - professore ordinario di Storia greca presso l'ateneo di Padova - vi aggiunge un punto interrogativo, quasi a premessa della questione che percorre le pagine del volume. Il tema non è nuovo per l'autrice, dal momento che si era già occupata in passato di questi temi'.

Il rapporto dell'Italia con la Grecia si costruì, tra Otto e Novecento a partire «[...] dalla storia antica, dalla scienza moderna, dalla politica estera e dallo sviluppo di un'identità nazionale incapace di fare i conti con il proprio passato»². Proprio questi squilibri nella costruzione identitaria furono fondamentali per l'interesse verso l'antichità, che veniva vissuta come manifestazione della grandezza perduta, riacquistabile recuperando i fasti del passato. Inutile dire che «la corrispondenza della romanità fascista col modello storico romano era in molti casi arbitraria, immaginaria, o semplicemente inesistente»3, ma su questo faceva leva il regime, nell'intento di creare un legame di continuità fra un passato glorioso e un futuro che si voleva altrettanto

\footnotetext{
${ }^{1}$ COPPOLA, Alessandra, Archaiologhía e propaganda: i greci, Roma e l'Italia, Roma, L'Erma di Bretschneider, 1995; BIASUTTI, Franco, COPPOLA, Alessandra (a cura di), Alessandro Magno in età moderna, Padova, CLEUP, 2009. Ha inoltre pubblicato: COPPOLA, Alessandra, Demetrio di Faro : un protagonista dimenticato, Roma, L'Erma di Bretschneider, 1995; ID., Il re, il barbaro, il tiranno : poesia e ideologia in età ellenistica, Padova, Esedra, 2002; ID., L'eroe ritrovato: il mito del corpo nella Grecia classica, Venezia, Marsilio, 2008.

2 COPPOLA, Alessandra, Una faccia, una razza? Grecia antica e moderna nell'immaginario italiano di età fascista, Roma, Carocci, 2013, p. 13.

3 GENTILE, Emilio, Fascismo di pietra, Roma-Bari, Laterza, 2007, p. 52.
} 
luminoso; contemporaneamente, con il deteriorarsi dei rapporti tra regime fascista italiano e Grecia, l'idea di grecità in Italia portò ad un declassamento della Grecia antica - considerata sempre e comunque seconda per gloria a Roma - e a una rottura della continuità fra Greci antichi e greci moderni (questi ultimi non avrebbero avuto nulla in comune con i loro avi).

«Missione universale, forza di comando, stato: era questa la triade di principi che Roma consegnava al fascismo»4: in quest'ottica l'esaltazione di Roma doveva mettere in risalto il primato della civiltà latina su tutte le altre del Mediterraneo.

Il primo capitolo L’idea di Roma, l’idea di Grecia, illustra i fondamenti alla base della costruzione dell'idea di Grecia e di Roma in Italia e nel paese ellenico. Il primo paragrafo ripercorre la fortuna dell'idea di Roma nel periodo fascista: emerge come l'immaginario della romanità fosse strumentale alla visione politica dell'epoca e l'ideologia fascista avesse compiuto un'operazione di reinvenzione dell'identità romana; il secondo, invece, è un breve excursus nella storiografia ellenica sulla Grecia antica. Il paragrafo che segue è un'attenta disamina del giudizio storiografico sulla Grecia antica e sull'Impero macedone in Italia durante l'epoca fascista. Coppola evidenzia, a questo proposito, i casi di giustapposizione della figura di Alessandro Magno a quella di Mussolini; malgrado ciò, il tenore generale della storiografia fa emergere un costante confronto fra i Greci e Roma, il cui esito è a tutto vantaggio della seconda. A questo schieramento su una visione piattamente filoromana si affiancava, sottotraccia, anche se solo in alcuni, una predilezione "clandestina" per il mondo greco5.

Nel secondo capitolo - intitolato Degenerazione - prende in esame la teoria propugnata inizialmente da Jakob Fallmerayer per cui i Greci moderni non avrebbero avuto più nulla a che spartire con quelli antichi, corrotti, come erano stati, dalle invasioni slave e albanesi susseguitesi durante il Medioevo ${ }^{6}$.

Il terreno di confronto si estese dalla storiografia - furono infatti molti a sposare, e altrettanti a confutare, le teorie di Fallmerayer - alla biologia. La "degenerazione" del

4 ZUNINO, Pier Giorgio, Ideologia del fascismo. Miti, credenze, valori, Bologna, Il Mulino, 1995, p. 73.

5 Si veda, ad esempio il caso dell'archeologo Giulio Rizzo «[...] che solo nelle lettere private esprimeva distacco nei confronti dei fervori romani e una decisa preferenza per il mondo greco, ma nei contributi a stampa rendeva comunque a Roma il posto che ufficialmente le spettava». COPPOLA, Alessandra, Una faccia, una razza?, cit., p. 43. Sul tema dell'atteggiamento degli intellettuali nei confronti della Grecia antica durante il periodo fascista, Dino Piovan osserva come: «In sintesi, gli studi di storia antica videro una divaricazione tra storia romana e storia greca, l'una infestata oltremisura dalla retorica e dalla propaganda, l'altra depressa ed emarginata anche nell'assegnazione delle cattedre universitarie». PIOVAN, Dino, Il fascismo e la storia greca, in BASSI, Jacopo, CANÈ, Gianluca, Sulle spalle degli antichi. L'immaginario della romanità e della grecità in età contemporanea, Milano, Unicopli, 2014, pp. 25-38, p. 26.

${ }^{6}$ Esposta in FALLMERAYER, Jakob Philip, Geschichte der Halbinsel Morea während des Mittelalters, Stuttgart-Tübingen, J.G. Cotta'schen Buchhandlung, 1830. 
popolo greco divenne quindi un tema oggetto di dibattito: in ambito italiano, come del resto a livello internazionale, le disquisizioni di antropologi e medici eugenetici si concentrarono, ad esempio, sull'individuazione delle cause della corruzione etnica alla base della caduta dell'Impero romano. Coppola prende in esame il dibattito razzista sviluppatosi intorno a una presunta «razza italiana»e il crescendo dei motivi denigratori nei confronti della razza greca; il fenomeno crebbe lungo tutto il corso degli anni Trenta, fino a toccare l'apogeo durante gli anni immediatamente a ridosso della guerra.

Nel capitolo successivo, Grecia moderna e Roma fascista, Coppola, dopo avere passato in rassegna i rapporti diplomatici fra Italia e Grecia, si sofferma sull'idea negativa della Grecia antica diffusasi fra gli intellettuali italiani; in questo caso fu in particolare la storia e il ruolo giocato dall'Impero bizantino ad attirare le critiche maggiori (e più feroci)7.

Il quarto capitolo è una rassegna dei viaggi compiuti in Grecia da artisti, letterati, diplomatici e studiosi; l'intento è mettere in evidenza lo iato creatosi tra l'aspettativa dei viaggiatori e la realtà dei fatti. Una breve appendice è dedicata proprio al libro di Henri Miller, Il Colosso di Marussi ${ }^{8}$, diario di viaggio dello scrittore e della sua scoperta della Grecia moderna e di quella antica.

Nelle pagine dell'epilogo, Coppola si sofferma brevemente sulla ricezione greca delle mire espansionistiche italiane e su quanto sia rimasto - o rimanga - del retaggio dell'antichità nella Grecia contemporanea.

Il volume si sviluppa intorno ad un'idea chiave: i rapporti politici e diplomatici, durante gli anni Trenta e i primi anni del secondo conflitto mondiale, influenzarono pesantemente lo studio dell'antichità classica e, in misura ancora maggiore, la percezione dell'immagine della grecità.

Il maggior pregio del volume è quello di cercare di ricostruire l'evoluzione dell'idea di grecità in Italia, in particolar modo durante l'epoca fascista: l'analisi dei testi e degli autori è quantitativamente e qualitativamente rilevante. Purtroppo la comparazione con l'immagine della grecità nel paese ellenico è solo abbozzata e non permette un confronto perfettamente speculare.

Il lavoro è certamente un punto di riferimento per lo studio di questo tema; gli spunti di ricerca che vengono offerti dall'autrice consentono di avvicinarsi all'argomento anche al lettore non specializzato.

7 COPPOLA, Alessandra, Una faccia, una razza, cit., pp. 91-93.

${ }^{8}$ MILLER, Henry, Il colosso di Marussi, Milano, Feltrinelli, 2007. 


\section{* L'autore}

Jacopo Bassi ha conseguito la laurea in Storia contemporanea (Università degli Studi di Bologna). Nel corso dei suoi studi si è occupato di storia della Grecia e dell'Albania in età contemporanea e di storia e istituzioni della Chiesa ortodossa. Ha lavorato per le case editrici Éditions des femmes, Il Mulino e Zanichelli.

URL: < http://www.studistorici.com/2009/o2/24/jacopo_bassi/ >

\section{Per citare questo articolo:}

BASSI, Jacopo, «Recensione: Alessandra COPPOLA, Una faccia una razza? Grecia antica e moderna nell'immaginario italiano di età fascista, Roma, Carocci, 2013, 168 pp.», Diacronie. Studi di Storia Contemporanea : Le città di Babele, 29/3/2015,

URL:< http://www.studistorici.com/2015/3/29/bassi_numero_21/ >

\section{Diacronie Studi di Storia Contemporanea www.diacronie.it}

Risorsa digitale indipendente a carattere storiografico. Uscita trimestrale. redazione.diacronie@hotmail.it

Comitato di redazione: Jacopo Bassi - Luca Bufarale - Elisa Grandi - Deborah Paci - Fausto Pietrancosta - Matteo Tomasoni - Luca Zuccolo

CC (i) $\odot$ Diritti: gli articoli di Diacronie. Studi di Storia Contemporanea sono pubblicati sotto licenza Creative Commons 2.5 . cc Possono essere riprodotti a patto di non modificarne i contenuti e di non usarli per fini commerciali. La citazione di EY NC ND estratti è comunque sempre autorizzata, nei limiti previsti dalla legge. 